\title{
Migraine with benign episodic unilateral mydriasis
}

This article was published in the following Dove Press journal:

International Journal of General Medicine

29 June 2011

Number of times this article has been viewed

\author{
Nedaa Skeik' \\ Fadi I Jabr ${ }^{2}$ \\ 'Mayo Clinic, Rochester, MN, USA; \\ ${ }^{2}$ Horizon Medical Center, Hospital \\ Medicine, Dickson, TN, USA
}

Correspondence: Nedaa Skeik Vascular Department, Mayo Clinic, 200 First Street SW Rochester, MN. 55905, USA

Tel +l 2073445 I54

Fax +I 5072661617

Email skeik.nedaa@mayo.edu
Abstract: Pupil asymmetry or anisocoria can have benign or malignant causes, and be categorized as acute or chronic. It can also be a normal finding in about $20 \%$ of cases. Benign episodic unilateral mydriasis is an isolated benign cause of intermittent pupil asymmetry. The exact pathophysiology is not always understood. According to one hypothesis, it is due to discordance between the sympathetic and parasympathetic systems. It is occasionally seen in patients with migraine. Some authors consider it a limited form of ophthalmoplegic migraine. We report a case of benign episodic unilateral mydriasis diagnosed in a 30-year-old lady with a history of migraine who had extensive negative neurological evaluation.

Keywords: anisocoria, migraine, unilateral episodic mydriasis

\section{Introduction}

Benign episodic unilateral mydriasis (BEUM) is an isolated cause of intermittent pupil asymmetry that might be caused by hyperactivity of the sympathetic nervous system or hypoactivity of the parasympathetic system. An association between BEUM and migraine has been suggested in the literature. Our case report of BEUM diagnosed in a young lady with migraine adds to the literature suggesting such an association.

\section{Case report}

A 30-year-old lady with a history of migraine headache presented with a one-year history of chronic intermittent, bilateral, throbbing, and at times very severe, headache that often lasted for days and was associated with blurry vision, nausea, vomiting, photophobia, and phonophobia. Severe episodes were sometimes associated with transient right eye mydriasis (Figure 1), significant lower extremity weakness and at times confusion that all resolved with the resolution of her migraine episode. She denied any recent head trauma, fever, chills, or other complaints. Her past medical history was important for obesity, tobacco use, fibromyalgia, and depression. Her medications at presentation were duloxetine $\mathrm{HCl} 60 \mathrm{mg}$ daily, trazodone $\mathrm{HCl}$ $100 \mathrm{mg}$ at night, gabapentin $900 \mathrm{mg}$ three times a day, and clonazepam $0.5 \mathrm{mg}$ twice a day as needed. She denied using illicit drugs, herbal, or other medications, including ones over the counter. There was no family history of similar eye disturbance. Vital signs were normal. Physical examination revealed a fixed dilated right pupil (Figure 1), decreased proximal and distal muscular strength $(3 / 5)$ in the lower extremities with normal reflexes. The rest of the neurological and physical examination 


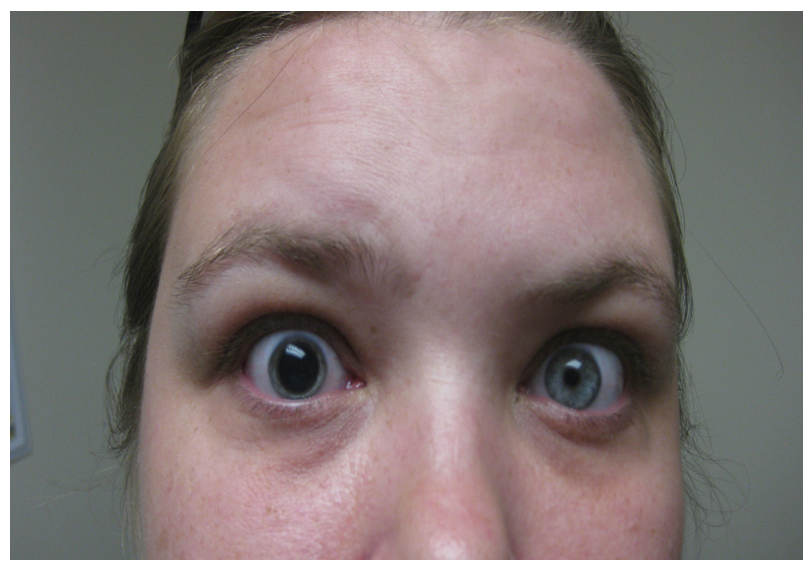

Figure I Intermittent right pupil dilation.

was unremarkable. Her complete blood count, metabolic profile, and thyroid function test were unremarkable. She had normal cerebrospinal fluid analyses, head and neck magnetic resonance imaging and angiography, cervical, thoracic, and lumbar magnetic resonance imaging, electroencephalography, and electromyography of the lower extremities.

Based on her presentation, laboratory and test results, she was diagnosed with an unusual case of migraine with benign episodic unilateral mydriasis. She was started on divalproex sodium, initially $500 \mathrm{mg}$ then increased to $1000 \mathrm{mg}$ daily, which led to significant improvement of her migraine headache as well as pupil disturbance.

\section{Discussion}

Causes of pupil asymmetry (anisocoria) range in seriousness from a normal, physiological condition (seen in $20 \%$ of normal people) to one that is immediately life-threatening, as seen with major stroke or intracranial bleed. Other causes include medications, infection, aneurysm, IIIrd nerve palsy, closed-angle glaucoma, and trauma. ${ }^{1,2}$

Benign episodic unilateral mydriasis is an isolated benign cause of pupil asymmetry. ${ }^{3}$ The episodes may be accompanied by blurred vision, orbital pain, headache, or photosensitivity. ${ }^{4}$ The underlying physiopathology is not always clear and may involve either parasympathetic deficiency or sympathetic hyperactivity affecting the iris. ${ }^{3,5}$ It can occasionally accompany migraine and some authors classify it as a limited form of ophthalmoplegic migraine. However, some cases have been described with no accompanying headache. ${ }^{3}$ The occurrence of mydriasis in ophthalmoplegic migraine can be due to functional exhaustion of parasympathetic fibers running within the
IIIrd cranial nerve. ${ }^{6}$ Other possible mechanisms include ischemia or oculomotor nerve demyelination caused by neuropeptides secreted at the level of circle of Willis upon the activation of the trigeminovascular system causing edema and inflammation. ${ }^{7}$

According to one report of seven patients with BEUM associated with migraine, four had classic, one had common, and one had post-traumatic migraine. BEUM was also reported in migraine without aura or ophthalmoplegia, suggesting that it is a concomitant symptom. ${ }^{89}$ Furthermore, a family history of migraine or headache was reported in patients with BEUM.,8

A detailed medical history, including active medications, full physical examination, including a careful ophthalmic and neurological system evaluation, and if indicated, imaging studies, should be performed to rule out other possible underlying conditions that sometimes can be lifethreatening. Management and prognosis are determined by the underlying etiology. According to one case series report, patients with isolated benign episodic mydriasis appear to have a benign neurological prognosis, and do not require further neurodiagnostic studies. ${ }^{5}$

Here we report a rare case of BEUM diagnosed in a young lady with a history of migraine, supporting the association between the two conditions. We call for more research to understand fully the underlying pathophysiology which would help in managing this condition.

\section{Disclosure}

The authors report no conflicts of interest in this work.

\section{References}

1. Biousse V, Newman NJ. Neuro-Ophthalmology Illustrated. Stuttgart, Germany: Thieme Verlag; 2009.

2. Kawasaki A. Disorders of pupillary function, accommodation and lacrimation. In: Miller NR, Newman NJ, Biousse V, Kerrison JB, editors. Walsh and Hoyt's Clinical Neuro-Ophthalmology. 6th ed. Baltimore, MD: Williams \& Wilkins; 2005.

3. Balaguer-Santamaria JA, Escofet-Soteras C, Chumbe-Soto G, Escribano-Subias J. Episodic benign unilateral mydriasis. Clinical case in a girl. Rev Neurol. 2000;31:743-745. Spanish.

4. Jacobson DM. Benign episodic unilateral mydriasis. Clinical characteristics. Ophthalmology. 1995;102:1623-1627.

5. Johnston JA, Parkinson D. Intracranial sympathetic pathways associated with the sixth cranial nerve. J Neurosurg. 1974;40:236.

6. Edelson RN, Levy DE. Transient benign unilateral pupillary dilation in young adults. Arch Neurol. 1974;31:12-14.

7. Bek S, Genc G, Demirkaya S, Eroglu E, Odabasi Z. Ophthalmoplegic migraine. Neurologist. 2009;15:147-149.

8. Woods D, O'Connor PS, Fleming R. Episodic unilateral mydriasis and migraine. Am J Ophthalmol. 1984;98:229-234.

9. Evans RW, Jacobson DM. Transient anisocoria in migraineur. Headache. 2003;43:416-418. 
International Journal of General Medicine

Dovepress

\section{Publish your work in this journal}

The International Journal of General Medicine is an international, peer-reviewed open-access journal that focuses on general and internal medicine, pathogenesis, epidemiology, diagnosis, monitoring and treatment protocols. The journal is characterized by the rapid reporting of reviews, original research and clinical studies across all disease areas.
A key focus is the elucidation of disease processes and management protocols resulting in improved outcomes for the patient. The manuscript management system is completely online and includes a very quick and fair peer-review system. Visit http://www.dovepress.com/ testimonials.php to read real quotes from published authors.

Submit your manuscript here: http://www.dovepress.com/international-journal-of-general-medicine-journal 